\title{
Use of temporary safety sutures during intrascleral fixation of intraocular lens
}

\author{
Tommy CY Chan ${ }^{1,2}$, FRCS, Alex $\mathrm{LK} \mathrm{Ng}^{3}$, FRCS, Arthur CK Cheng ${ }^{2}$, FRCS \\ ${ }^{1}$ Department of Ophthalmology and Visual Sciences, The Chinese University of Hong Kong, Hong Kong \\ ${ }^{2}$ Department of Ophthalmology, Hong Kong Sanatorium and Hospital, Hong Kong \\ ${ }^{3}$ Hong Kong Ophthalmic Associates, Hong Kong
}

Correspondence and reprint requests:

Dr Arthur CK Cheng, Department of Ophthalmology, Hong Kong Sanatorium and Hospital, 4/F, Li Shu Fan Block, 2 Village Road, Happy Valley, Hong Kong.

Email:drarthurcheng@gmail.com

Sutureless intrascleral intraocular lens (IOL) implantation is an advanced scleral fixation technique to eliminate the suture-related complications of erosion and exposure after conventional methods. ${ }^{1}$ Nonetheless, it is technically challenging. Crushing and pulling of the haptic of a threepiece IOL may cause deformation or breakage and hence tilting or dropping of the IOL. We introduce an additional step to enhance safety of the procedure.

Two additional $1.0-\mathrm{mm}$ incisions are made at the peripheral cornea at 2 and 8 o'clock positions (Figure a). With the aid of a hook, a 10-0 nylon suture is inserted through each of the $1.0 \mathrm{~mm}$ paracenteses to form two suture loops in the anterior chamber (Figure b). The suture loop from the 8 o'clock incision is then externalized through the superior incision (Figure c). During insertion of the IOL injector, the cartridge is inserted through the externalized suture loop (Figure d). The leading haptic is then passed through the suture loop at the 2 o'clock incision during the injection process (Figure e ). A pair of forceps is used to tighten the suture loop. After removal of the IOL injector, the trailing haptic is kept outside the eye to prevent the IOL from falling into the vitreous cavity. The trailing haptic is then entwined by the externalized suture loop originating from the 8 o'clock incision (Figure f). A pair of forceps is used to pull the suture loop back into the eye securing the hapticoptic junction of the trailing end (Figure $\mathbf{g}$ ), followed by externalization of the leading and trailing haptics through the sclerotomy (Figures h to j). The two safety sutures enhance the stability of the IOL. After fixation of the externalized haptics, the two suture loops are removed.
The use of safety sutures during intrascleral IOL fixation is very helpful. In one case, the leading haptic was accidentally inserted posterior to the iris plane after the IOL was released from the injector. The IOL was prevented from dropping posteriorly by the safety suture at the 2 o'clock incision, which also aided manipulation of the leading haptic back to the anterior chamber. In another case, the leading haptic was bent and could not be inserted into the needle for externalization when performing the Yamane technique. ${ }^{2}$ With the aid of the safety sutures, the IOL was stabilized in the anterior chamber while the bent leading haptic was rotated out of the main wound for trimming. The use of safety sutures is particularly helpful in cases of well-dilated pupil and aniridia.

In a series of 208 eyes undergoing sutureless scleral fixated IOL, intraoperative haptic breakage and deformation occurred in one and two eyes, respectively. ${ }^{3}$ Only one of eight eyes undergoing sutureless scleral fixation had haptic breakage. ${ }^{4}$ Manipulation of the trailing haptic can be difficult after the leading haptic is externalized. The leading haptic may rotate and slip back into the eye. Three cases of intraoperative haptic dislocation using the transconjunctival trochar-assisted technique have been reported. ${ }^{5}$ The trailing haptic may be damaged when it becomes the only pivot point being held by the forceps to prevent dropping of the IOL posteriorly. A push-and-pull hook technique has been described to facilitate manipulation of the trailing haptic. ${ }^{6}$ Keeping the leading haptic and holding needle inside the eye is suggested to facilitate externalization of the trailing haptic. ${ }^{2}$ 
(a)

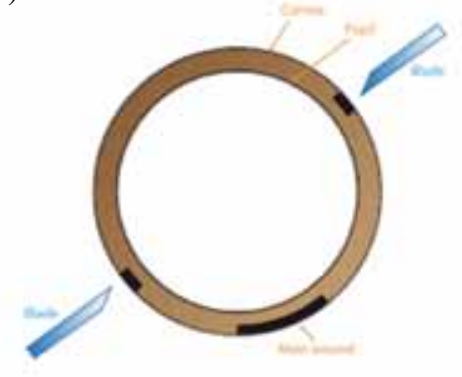

(d)

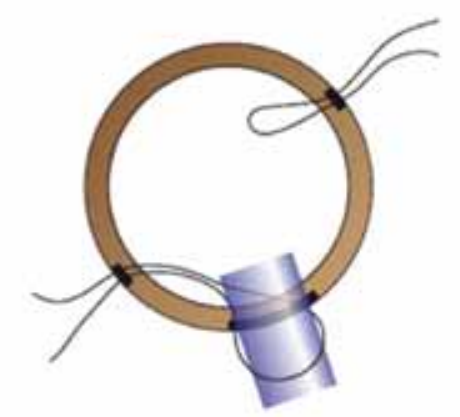

(g)

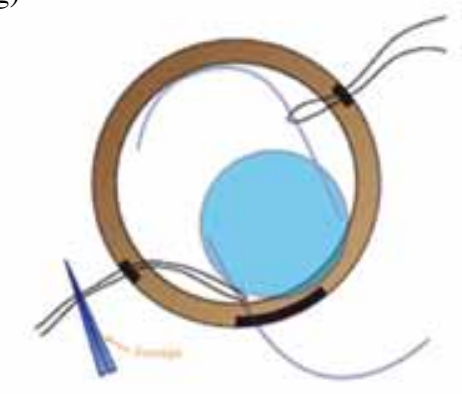

(j)

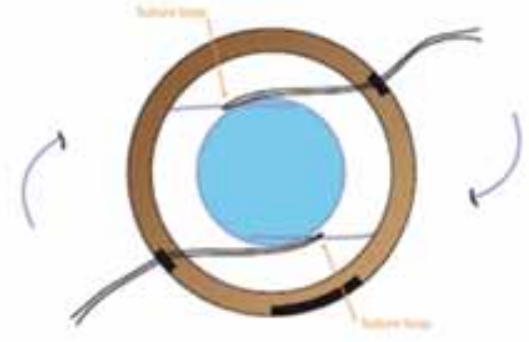

(b)

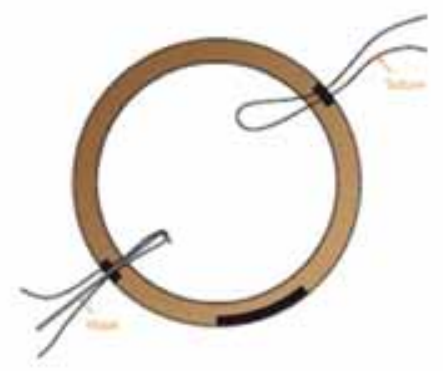

(e)

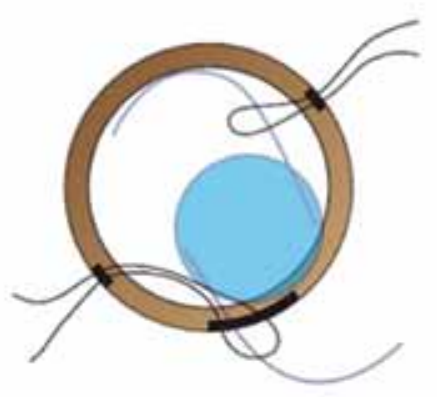

(h)

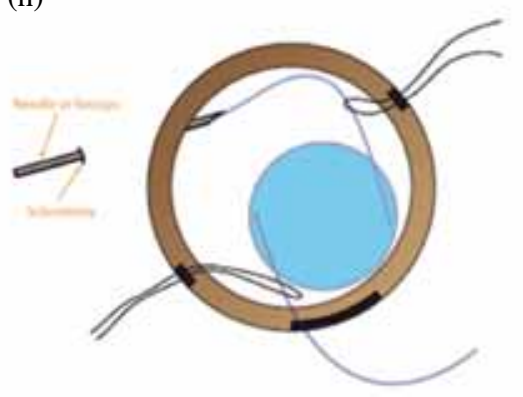

(c)

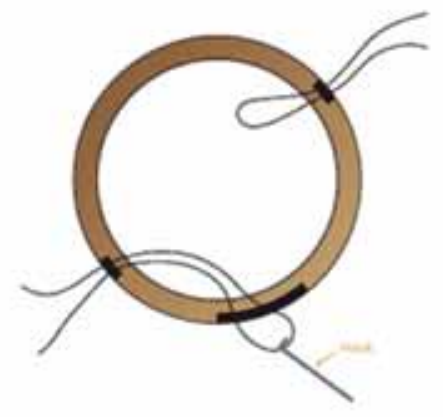

(f)

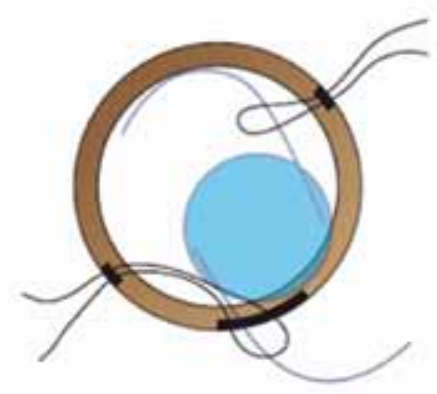

(i)

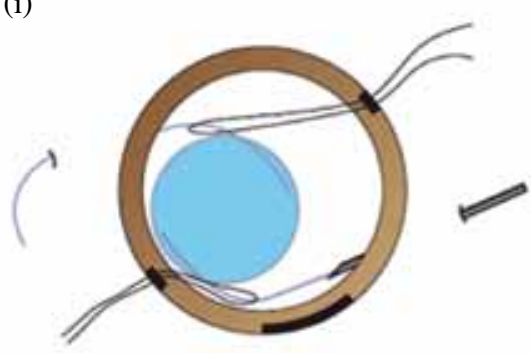

Figure. (a) Two 1.0-mm incisions are created at 2 and 8 o'clock positions. (b) Threading of two 10-0 monofilament nylon suture loops into the anterior chamber. (c to g) Formation of suture loop around the leading and trailing haptics during intraocular lens insertion. (h to j) Externalization of the haptics through sclerotomies.

Sutureless intrascleral fixated IOL implantation is increasingly popular among anterior segment surgeons. The IOL should stay within the sulcus and pars plana during the procedure after thorough anterior vitrectomy. Manipulation of the IOL can be stressful, especially when vitreoretinal support is not available. We added a maneuver to help stabilize the IOL. The technique is versatile and can be incorporated into various sutureless scleral fixation techniques and simple because it involves only the threading of two temporary sutures without tying knots. Although the use of safety sutures may be unnecessary for experienced surgeons, we recommend its use by trainees to enhance the safety of the procedure.

\section{Declaration}

The authors have no conflicts of interest to disclose. 


\section{References}

1. Karadag R, Celik HU, Bayramlar H, Rapuano CJ. Sutureless intrascleral fixated intraocular lens implantation. J Refract Surg 2016;32:586-97.

2. Yamane S, Sato S, Maruyama-Inoue M, Kadonosono K. Flanged intrascleral intraocular lens fixation with doubleneedle technique. Ophthalmology 2017;124:1136-42.

3. Kumar DA, Agarwal A, Packiyalakshmi S, Jacob S, Agarwal A. Complications and visual outcomes after glued foldable intraocular lens implantation in eyes with inadequate capsules. J Cataract Refract Surg 2013;39:1211-8.
4. Saleh M, Heitz A, Bourcier T, et al. Sutureless intrascleral intraocular lens implantation after ocular trauma. J Cataract Refract Surg 2013;39:81-6.

5. Abbey AM, Hussain RM, Shah AR, Faia LJ, Wolfe JD, Williams GA. Sutureless scleral fixation of intraocular lenses: outcomes of two approaches. The 2014 Yasuo Tano Memorial Lecture. Graefes Arch Clin Exp Ophthalmol 2015;253:1-5.

6. Ohta T, Toshida H, Murakami A. Simplified and safe method of sutureless intrascleral posterior chamber intraocular lens fixation: Y-fixation technique. J Cataract Refract Surg 2014;40:2-7. 